\title{
Tendencias sobre la contabilidad ambiental en el campo de la investigación en los últimos cinco años
}

\section{Trends on environmental accounting in the field of research in the last five years}

Ernesto Leonel Chávez H. ${ }^{1}$

Lina M. Lugo Gómez ${ }^{2}$

\section{Artículo de investigación}

Recepción: 05-04-2021

Aceptación: 20-08-2021

El Conuco es una revista de acceso abierto revisada por pares. (C) $2018 \mathrm{El}$ autor (es). Este es un artículo de acceso abierto distribuido bajo los términos de la Licencia Internacional Creative Commons Attribution 4.0 (CC-BY 4.0), que permite el uso, distribución y reproducción sin restricciones en cualquier medio, siempre que se acredite el autor y la fuente originales.

Consulte http://creativecommons.org/licenses/by/4.0/. ऽOPEN ACCESS

\section{(c) $(\$)$}

Magíster en Desarrollo Sostenible y Medio Ambiente (Universidad de Manizales), Economista (Universidad Católica de Colombia); Profesor de planta de la Universidad de los Llanos. Correo electrónico institucional: echavez@ unillanos.edu.co, ORCID: ID https://orcid.org/0000-00023008-0502

2 Estudiante de VI semestre, programa de Contaduría Pública, Facultad Ciencias Económicas, Universidad de los Llanos, correo institucional: lina.lugo@unillanos.edu.co, ORCID: ID https://orcid.org/0000-0003-1694-3276

Este artículo hace parte del trabajo de aula del curso de Metodología de la Investigación diurno del programa de Contaduría Pública

Como citar este artículo / To reference this articcle: M., (2016). Tendencias sobre la contabilidad ambiental en el campo de la investigación en los últimos cinco años EL CONUCO: (investigación, economía y sociedad), 3(2), pp. 1-8.

DOI: https://doi.org/10.22579/2619-614X.777

\section{Resumen}

El afán por investigar acerca de la contabilidad ambiental, ha llevado a varios investigadores por develar conocimiento con relación a este campo de la contabilidad. El objetivo principal de la investigación es determinar las tendencias y avances de la contabilidad ambiental en el escenario de la investigación a nivel nacional e internacional. El enfoque investigativo propuesto es cualitativo descriptivo; para el propósito de recabar la información se diseñan y aplican un formato de análisis de contenido, para la identificar, clasificar y examinar los datos cualitativos obtenidos de las bases de datos de revistas indexadas y repositorios.

Palabras clave. Medio ambiente, costos, control, desarrollo sostenible.

Códigos JEL: O13, Q52, Q01.

\section{Abstract}

The eagerness to investigate about environmental accounting has led several researchers to unveil knowledge in relation to this field of accounting. The main objective of the research is to determine trends and advances in environmental accounting in the research scenario at the national and international level. The proposed research approach is qualitative descriptive; For the purpose of gathering the information, a content analysis format is designed and 
applied to identify, classify and examine the qualitative data obtained from the databases of indexed journals and repositories.

Keywords. Environment, costs, control, sustainable development.

\section{Introducción}

Actualmente el medio ambiente es importante, pues es necesario reconocer que se están agotando los recursos naturales a nivel mundial y en el campo empresarial los profesionales, empresarios y la sociedad en general tienen la obligación de adoptar una responsabilidad ambiental, es imprescindible crear estrategias que permitan evitar y disminuir el impacto negativo que el uso desmedido de los recursos puede generar.

Si bien, La contabilidad ambiental comenzó a recibir atención durante la crisis energética de 1970, no fue hasta la década de 1990 con la aparición del activismo de protección del medio ambiente, que tomo total relevancia en el marco corporativo. La contabilidad ambiental surgió de la necesidad de cuantificar y controlar los impactos ambientales ocasionados en el medio ambiente por la actividad empresarial, y la necesidad de prevenir o corregir dichos impactos. Desde comienzos de la década de los setentas, se empezaron a desarrollar estudios de contabilidad ambiental en dos niveles: el académico y el institucional, como resultado de estos estudios surgieron propuestas para incorporar las consideraciones ambientales en la medición de indicadores de bienestar y sostenibilidad.

Algunos estudios realizados mencionan la relación entre el impacto ambiental y los procesos de depreciación y medición de la producción, en este sentido Solow (1986, como se citó en Casado y Santamaría, 2003), destaca que:

En el que amplía el concepto de producto nacional neto para incorporar la depreciación de los recursos no renovables. Hartwick (1990), incorpora en la medición de la producción neta, la depreciación de los recursos renovables y los costes de reducción de la contaminación. (p.4)

Es decir a partir de los diversos tratados y estudios que se han preocupado por el impacto que genera la producción empresarial al medio ambiente, se dio inicio a una nueva era en donde se tiene en cuenta la reducción de este impacto mediante instrumentos como el tratado de Kioto, el cual tiene como principal objetivo que los países participantes mediante normas, estándares internacionales, certificaciones, reduzcan la emisión de gases de efecto invernadero, y produzcan proyectos relacionados al desarrollo limpio, entre otros. Así mismo, la norma ISO 26000 les ayudará en su esfuerzo por operar de la manera socialmente responsable que la sociedad exige cada vez más (ISO, 2010).

La finalidad de este proyecto, es describir las tendencias encontradas en el análisis de la información obtenida acerca de contabilidad ambiental y determinar los avances de esta en el escenario de la investigación a nivel nacional e internacional.

\section{Aproximación al marco teórico y conceptual}

La contabilidad ambiental es un sistema que resalta la contribución de los recursos económicos obtenidos por la explotación y uso de recursos naturales, o bien pueden ser los costos o pérdidas en los que incurre por el desgaste, contaminación o impactos ocasionados al medio ambiente por la actividad económica de la entidad.

Para soportar este análisis se tienen en cuenta teorías y paradigmas como: Economía de frontera, teoría tridimensional de la contabilidad y teoría del desarrollo sostenible; que reflejan la impor- 
tancia y lo relevante que es el medio ambiente en el ámbito social y económico, como lo menciona, Pisch (2002 citado en Miranda, et al 2007), que:

El concepto de desarrollo sostenible concibe el desarrollo como un proceso armónico, donde la explotación de los recursos, la dirección de las inversiones, la orientación del cambio tecnológico y las transformaciones institucionales deben corresponderse con las necesidades de las generaciones presentes y futuras. Así, se presenta el desarrollo como un proceso que requiere un progreso global, tanto en materia económica y social como en los órdenes ambiental y humano. (párr. 18).

Esto genera una responsabilidad social ambiental debido que el medio ambiente ha otorgado múltiples recursos y beneficios que a lo largo de la historia han sido usados como herramienta de producción, la teoría de desarrollo sostenible demuestra que la naturaleza es un importante benefactor de recursos, Martínez y Figueroa (2013), afirman que en la década de los sesenta la economía de frontera:

Posicionaba el uso de la naturaleza como herramienta para el beneficio humano, y relega el ambiente a una condición de objeto manipulable a conveniencia sobre el cual se pueden aplicar las estrategias que se requirieren para fomentar el acopio y concentración material como ideal de calidad de vida. (p.4).

Si bien, el medio ambiente ha sido generoso con el ser humano y con su actividad económica, esto ha derivado una responsabilidad ambiental y social que traspasa fronteras pues el medio ambiente es el hogar del ser humano; y este se ha esforzado por optimizar los beneficios otorgados y sacar el mayor provecho de estos, "cuando una actividad hace surgir amenazas de daño para el medio ambiente o la salud humana, se deben to- mar medidas de precaución incluso si no se han establecido de manera completamente científica algunas relaciones de causa-efecto." (Cózar, 2005 , p. 137). De allí, se hace necesario fomentar su conservación, obligando a las compañías y aquellos que desarrollen actividades económicas que intervengan en el medio ambiente a hacerse responsable de la protección de este y garantizar su buen uso.

Como se mencionó, la contabilidad ambiental empresarial es significativa y ha proporcionado importantes beneficios tanto en el ámbito social como en actividades comerciales, permitiendo que de allí surgiera la necesidad de controlar estos recursos y revelarlos en la información económica de las entidades que permitan compararlos con otros y revelar de forma real la situación social y ambiental de estas. La teoría tridimensional "permite a través del método deductivo construir los componentes tecno-conceptual y tecno-procedimental de los sistemas contables, como lineamientos normalizadores y guías en el proceso de reconocimiento, medición-valoración, presentación y revelación de información contable" (Mejía, et al, 2014, p.97), esto con el fin de que la información compartida con los usuarios sea lo más verídica, coherente y concisa. Reconociendo que el contador público y el empresario tienen una responsabilidad social ambiental y deben considerar en el ejercicio de su actividad económica velar por la preservación de los recursos ambientales.

El medio ambiente, en el marco económico pasa a un segundo plano cuando de negocios se trata, pues las empresas se encuentran más interesadas en la utilidad que puedan obtener dejando de lado la importancia de conservar los recursos, "el nivel de desarrollo se mide de forma equivoca, pues se evalúa solo desde el contexto económico dejando de lado el social y ambiental"(Mantilla, 
et al, 2015, p.138), son muy pocas las entidades que se comprometen con el cuidado y buen uso de los recursos naturales, debido que, este compromiso ambiental puede aumentar los costos y/o gastos en su actividad económica, lo cual representaría una disminución de sus utilidades.

Por lo tanto, son muy pocas las empresas que implementan políticas contables responsables con el medio ambiente dentro de su organización, tal y como lo señala Sánchez (2015), asegura que:

Fortalecer y difundir la contabilidad ambiental, requiere incorporar la responsabilidad medioambiental al sistema económico del mercado, en el cual se combinen instrumentos de mercado y acciones preventivas y restauradoras, que permitan la cuantificación económica de las acciones necesarias para mantener el equilibrio ambiental, de tal manera que se puedan trasladar los costos implícitos hacia la empresa, en consecuencia, la contabilidad ambiental debe estar en capacidad de evidenciar estas circunstancias. (p.9)

Razón por la cual, la norma ISO 14001 (2015), "proporciona a las organizaciones un marco con el que proteger el medio ambiente y responder a las condiciones ambientales cambiantes, siempre guardando el equilibrio con las necesidades socioeconómicas" (p.2). Igualmente, la ONU creó el Comité de las Naciones Unidas de Expertos sobre Contabilidad Ambiental y Económica (UNCEEA), que tiene como objetivo efectuar una estrategia global que mitigue los daños ocasionados al medio ambiente y que las compañías se acoplen a este sin ninguna dificultad.

En concordancia con lo anterior, es importante reconocer los principales factores que tienen en cuenta las compañías para implementar políticas contables responsables con el medio ambiente. Por ende, se hace necesario contar con instrumentos de medida, análisis y controles más precisos y objetivos, para así reconocer, subsanar o mitigar el daño que causa al medioambiente concurrido con el hecho económico, creando estrategias especiales, con el fin de añadir valor a la compañía y que estas adquieran un compromiso responsable con el medio ambiente, como mencionan Sánchez y Rodríguez (2017), indican que: "En los países industrializados, se ha venido realizando actividades en las empresas para mitigar los costos ambientales, y es necesario que todos los usuarios y consumidores se comprometan." (p.26); es importante que los usuarios reconozcan que tienen una obligación social con el medio ambiente y que este compromiso puede generarles beneficios que les permite tener una actividad empresarial responsable.

De igual manera se menciona que el problema no se limita a cierta área geográfica o en un periodo en específico, debido a que el impacto ambiental se puede presentar en cualquier espacio geográfico contaminado por la liberación de materiales, residuos extraños o aleatorios, que no fueron remediados oportunamente y siguen causando efectos negativos que se convierten en un problema ambiental proveniente de una actividad económica existente, que en su condición, genera frente a terceros un riesgo permanente y potencial para la salud, el ecosistema y la propiedad.

\section{Marco legal y normativo.}

Como se ha mencionado, la contabilidad ambiental es un tema que le compete a toda la sociedad en general, en este caso, es importante recalcar la responsabilidad que tiene el empresario en este ámbito y como los gobiernos a nivel nacional e internacional están controlando el uso de los recursos naturales en la explotación económica. En cuanto a esto, los gobiernos tienen la obligación de ejercer un control frente a este escenario, para ello, 
el Congreso Nacional de Colombia creó la ley 99 de 1993, en la cual: "crea el Ministerio del Medio Ambiente, se reordena el Sector Público encargado de la gestión y conservación del medio ambiente y los recursos naturales renovables, se organiza el Sistema Nacional Ambiental, SINA y se dictan otras disposiciones". Este ministerio tiene como objetivo armonizar el respeto, regular el manejo, conservación y protección del medio ambiente, asegurando a cualquier ciudadano colombiano la oportunidad de gozar de un ambiente sano.

Por otra parte en Colombia la ley 1314 de 2009 , regula los principios y normas de la contabilidad financiera; estos principios obligan a las empresas y preparadores a presentar información transparente, confiable y comparable con los estándares internacionales incluyendo las mejores prácticas del desarrollo socioeconómico, las empresas tienen la obligación de reconocer el impacto que su actividad económica ocasiona en el medio ambiente, pues estos daños ocasionados pueden incurrir en futuros costos, demandas, sanciones, entre otros.

\section{Marco contextual}

Para el desarrollo de esta propuesta se revisaron veinte artículos de investigación que tienen como objeto de estudio paradigmas contables, su evolución cronológica; conceptos relacionados con gestión ambiental, políticas y tendencias de investigación para el desarrollo de normativas que regulen el beneficio, uso y conservación de los recursos ambientales.

Aun así, algunas de las empresas que hacen uso de los recursos naturales no tienen conciencia de su responsabilidad ambiental y que al mismo tiempo son responsables con la sociedad por el mal uso que se le da al medio ambiente, por tal motivo, se hace necesario el reconocimiento ambiental "en la gestión de sus actividades, las empresas practican y están sujetas a una secuencia de situaciones de naturaleza ecológica que se refleja en sus activos de diferentes maneras" (Gomes, et al, 2020, p.20). Por ello, la creación de políticas amigables con el medio ambiente, permite el uso responsable de la naturaleza mientras el empresario hace uso de esta para el desarrollo de su actividad económica.

Este proyecto es una producción intelectual fruto de la investigación que se visibiliza en las bases de datos.

\section{Método}

La metodología utilizada para la elaboración de esta propuesta es de tipo cualitativo, definiendo "la metodología cualitativa como la investigación que produce datos descriptivos: las propias palabras de las personas, habladas o escritas, y la conducta observable" (Quecedo y Castaño, 2002, p. 7), Porque pretende establecer las cualidades de la información obtenida de fuentes secundarias, razón por la cual se recopilo una serie de trabajos escritos en manera de trabajos de grado y artículos de investigación que tengan como objeto de estudio, contabilidad, gestión ambiental empresarial y contabilidad ambiental.

De igual forma, este estudio se enmarca en una investigación de carácter descriptivo, puesto que con este proyecto "se busca especificar las propiedades, las características y los perfiles de procesos, objetos o cualquier otro fenómeno que se someta a un análisis" (Hernández, et al, 2014, p.92), debido que, el propósito de este proyecto es describir las tendencias en investigación sobre contabilidad ambiental, es importante reconocer sus principales características y los perfiles de investigación más importantes en cuanto al objeto de estudio que es contabilidad ambiental.

Hay que mencionar que, este proyecto se constituye en las fuentes secundarias como artículos de 
revistas indexadas, trabajos de grados, tesis, que se han desarrollado sobre el objeto de estudio, "una estructura categórica a partir de la cual podemos responder a las preguntas formuladas a un problema práctico así como a las preguntas de investigación" (Picón y Melian, 2014, p.103), que permitirá categorizar las fuentes consultadas, llevando un orden de estudio que en el proceso brindará respuestas claras a las preguntas generadas en el transcurso del desarrollo del proyecto, como Martínez(2006), afirma que: "Sería la nueva realidad que emerge de la interacción de las partes constituyentes, sería la búsqueda de esa estructura con su función y significado" (p. 132). Aquí el reafirma que la unidad de análisis seria esa nueva realidad que surge mediante el desarrollo del proyecto.

Como se ha dicho, el análisis de contenido es una herramienta que permite y guía al investigador hacia el entendimiento del objeto de estudio en el que se encuentra interesado mediante la comprensión de su comunicación, tal como lo indican, Hernández, Fernández y Baptista (2014), al hacer mención sobre las consideraciones de algunos autores que llegan a considerar al análisis de contenido como un diseño. Pero a pesar de la definición que se haga, realmente es una técnica útil para analizar distintos procesos de comunicación en variados contextos porque este instrumento se basa en el cómo se comunican los individuos en los diversos escenarios y situaciones; de esa interacción esta herramienta permite generar un análisis más cercano a la realidad.

\section{Reflexiones}

En definitiva, los empresarios, contadores públicos y la sociedad en general tienen la obligación de reconocer e incrementar en su ejercicio su responsabilidad ambiental, las empresas deben incrementar políticas contables que reconozcan estos hechos y que trabajen en pro de prevenir posibles daños a los ecosistemas, si bien, hoy en día no existen tantos proyectos que arrojen resultados claros sobre este objeto de estudio, los gobiernos han hecho esfuerzos para controlar las practicas que puedan ocasionar algún tipo de daño en el medio ambiente, aunque no son muchas las leyes existentes las compañías pueden usar modelos internacionales como las ISO que fueron especialmente creados para este fin.

De igual modo, esta responsabilidad también debe recaer en el ámbito académico, como lo afirman, Martínez y Sánchez (2019), indicando que: "la crisis ambiental y del desarrollo sostenible por parte de la contabilidad no es solo un problema técnico que se resuelve únicamente en el ámbito organizacional, sino que también el ámbito académico" (párr. 26), si bien es un tema importante, de orden social y económico, faltan proyectos de investigación que arrojen datos que hagan frente a esta problemática.

Conforme a lo descrito anteriormente, indiscutiblemente para lograr una cultura de responsabilidad ambiental es necesario informar y alertar al preparador y a los empresarios sobre los daños en los que puede incurrir su actividad económica al medio ambiente, es necesario presentar propuestas y/o estudios que no solo arrojen resultados sino que también ayuden al desarrollo de normativas y estrategias que mitiguen y eviten el impacto ambiental, por esta razón, se hace "evidente la necesidad de investigaciones críticas que puedan proponer soluciones al respecto en pueda sustentarse un desarrollo más ‘profundo de la Contabilidad Ambiental en los programas universitarios”(Quiñones, et al, 2016, p.163). 


\section{Referencias}

Casado, J. y Santamaría, M. (2003). Contabilidad ambiental: antecedentes internacionales. [Ponencia]. $V$ Reunión de Economía Mundial. Sevilla, España. https://idus.us.es/bitstream/handle/11441/97176/contabilidad_ambiental_antecedentes_internacionales. pdf? sequence $=1$ \&is Allowed $=y$

Cózar Escalante, J. M. d. (2005). Principio de precaución y medio ambiente. Revista española de salud pública, 79 (2), 133144. http://scielo.isciii.es/pdf/resp/ v79n2/colaboracion $1 . p d f$

Gomes, K., Blazquez, A., Leite, E. y González, G. (2020). Contabilidad ambiental desde una visión de responsabilidad social. International Journal of Professional Business Review (JPBReview), 5 (1), 17-27. https:/ / dialnet.unirioja.es/servlet/articulo?codigo $=7291664$

Hernández, R., Fernández, C. y Baptista, P. (2014). Metodología de la Investigación. McGraw-Hill. https://www.uca.ac.cr/ wp-content/uploads/2017/10/Investigacion.pdf

ISO 14001:2015. (2015, 15 de septiembre). Sistemas de gestión ambiental. Organización Internacional de Normalización. https://www.nueva-iso-14001.com/ pdfs/FDIS-14001.pdf

ISO 26000:2010. (2010, 03 de noviembre). Responsabilidad social. Organización Internacional de Normalización. https:/ / www.iso.org/files/live/sites/isoorg/ files/archive/pdf/en/iso_26000_project_overview-es.pdf
Ley 1314 de 2009. (2009, 13 de julio). Congreso de la Republica de Colombia. Diario oficial No. 47.409. http:/ /www. secretariasenado.gov.co/senado/basedoc/ley_1314_2009.html

Ley 99 de 1993. (1993, 22 de diciembre). Congreso de la República de Colombia. Diario oficial No. 41.146. http:// www.secretariasenado.gov.co/senado/ basedoc/ley_0099_1993.html

Mantilla, E., Cabeza, M. y Vargas, J. (2015). La realidad del desarrollo y la contabilidad ambiental. Saber, ciencia y libertad, 10 (2), 133-146. https://dialnet.unirioja.es/ servlet $/$ articulo? codigo $=5329131$

Martínez, J. y Figueroa, A. (2013). Evolución de los conceptos y paradigmas que orientan la gestión ambiental ¿cuáles son sus limitaciones desde lo glocal? Revista Ingenierías Universidad de Medellín, 13 (24), 13-27. http://www.scielo.org. co/pdf/rium/v13n24/v13n24a02.pdf

Martínez, M. (2006). La investigación cualitativa (síntesis conceptual). Revista IIPSI, 9 (1), 123-146. https:// sisbib.unmsm. edu.pe/bvrevistas/investigacion_psicologia/v09_n1/pdf/a09v9n1.pdf

Martínez, M. y Sánchez, A. (2019). Una mirada a la contabilidad ambiental en Colombia desde las perspectivas del desarrollo sostenible. Revista de Facultad de Ciencias Económicas, 27 (1), 87-106 http://www.scielo.org.co/ scielo.php?script $=$ sci_arttext\&pid $=$ S012168052019000100087\&lang=es 


\section{Referencias}

Mejía, E., Montilla, O., Montes, C. y Mora, G. (2014). Teoría tridimensional de la contabilidad T3C (versión 2.0): Desarrollos, avances y temas propuestos. Revista Libre Empresa, 11 (2), 95-120. https:// revistas.unilibre.edu.co/index.php/libreempresa/article/view/3027/2437

Miranda, T., Suset, A., Cruz, A., Machado, H., y Campos, M. (2007). El Desarrollo sostenible. Perspectivas y enfoques en una nueva época. Pastos y forrajes, 30 (2), 191-204. http://scielo.sld.cu/scielo.php?script=sci_arttext\&pid=S086403942007000200001\#: :text $=\mathrm{El} \% 20$ concepto $\% 20 \mathrm{de} \% 20$ desarrollo $\% 20$ sostenible, las $\% 20$ generaciones $\% 20$ presentes $\% 20 \mathrm{y} \% 20$ futuras

Naciones Unidas. (ONU) (2016). Sistema de contabilidad ambiental y económica 2012. https:// unstats.un.org/unsd/envaccounting/seeaRev/CF_trans/SEEA_ CF_Final_sp.pdf

Picón, D. Y Melian, Y. (2014). La unidad de análisis en la problemática enseñanza-aprendizaje. Informe Científico Técnico UNPA, 6 (3), 101-117. https:// dialnet.unirioja.es/servlet/ articulo?codigo $=5123550$
Quecedo, R. Y Castaño, C. (2002). Introducción a la metodología de investigación cualitativa. Revista de Psicodidactica, (14), 5-39. https://www.redalyc.org/ pdf/175/17501402.pdf

Quiñones, E., Balladares, K. y Estrada, F. (2016). Reflexiones sobre la Contabilidad Ambiental. Revista Publicando, 3 (7), 156-166. https://dialnet.unirioja.es/ servlet/articulo? codigo $=5833482$

Sánchez, H. y Rodríguez, C. (2017). Determinar los costos ocultos ambientales, de los residuos peligrosos en las empresas manufactureras de muebles para oficina. [Proyecto de grado, Corporación Universitaria Minuto de Dios]. Archivo digital. https://repository.uniminuto. edu/bitstream/handle/10656/5399/ UVD-TCP_SanchezRojasHarol_2017. pdf? sequence $=1$ \&isAllowed $=\mathrm{y}$

Sánchez, M. (2015). La contabilidad ambiental, equilibrio entre el crecimiento económico y preservación delmedio ambiente. Una minirevisión de literatura. (Trabajo de grado, Pontificia Universidad Javeriana). Archivo digital. https://repository.javeriana.edu. co/bitstream/handle/10554/16680/ SanchezMoraMiguelAlejandro2015. pdf ? sequence $=1$ \&isAllowed $=\mathrm{y}$ 